\title{
El arte rupestre de Laguna Miramar, Chiapas. Estilos y temporalidades
}

\author{
Rock art at Lake Miramar, Chiapas. Styles and temporalities
}

\author{
RAMÓN Folch GONZÁLEZ* \\ Josuhé Lozada Toledo** \\ doi.org/10.29043/liminar.v20i1.888
}

Resumen: En este trabajo se analiza el arte rupestre de la Laguna Miramar, ubicada en el municipio de Ocosingo, Chiapas, y se compara con manifestaciones similares en el área con el fin de refinar una tipología regional de estilos y pigmentos. El registro detallado de las pinturas y petrograbados se hizo con fotografía, DStrech y dibujo. Se propone asociar estilos y pinturas cercanos a cuerpos de agua a los periodos Preclásico Tardío, Postclásico e Histórico/Colonial. Igualmente se revisan los trabajos sobre arte rupestre en Chiapas, y particularmente en la Selva Lacandona. El esquema propuesto articula el estudio descriptivo para realizar interpretaciones respecto a temas como religión, paisaje y peregrinaciones.

Palabras clave: arte rupestre, Postclásico, petrograbados, pigmentos, lacandones, arqueología de lagos.

Abstract: This article analyses rock art at Lake Miramar, Ocosingo, Chiapas, and compares it to similar manifestations in the region in an attempt to perfect a typology based on styles and pigments. Paintings and rock carvings were documented using photographs, DStrech software, and drawings. We propose linking patterns in style and pigments, near bodies of water, to Late Preclassic, Postclassic, and Historic/ Colonial periods. We also outline and discuss the research on rock art in Chiapas and especially in the Lacandon Jungle. The proposed outline brings together various descriptive studies in order to interpret factors such as religion, landscape, and pilgrimage.

Key words: rock art, Postclassic, rock carvings, pigments, Lacandon, lake archaeology.

Recibido: 24 de agosto de 2020 Aprobación: 1 de junio de 2020 Publicación: 8 de octubre de 2021
* School of Human Evolution and Social Change, Arizona State University,

Tempe, AZ, Estados Unidos rfolch@asu.edu

D 0000-0003-0709-2721 **Dirección de Estudios Arqueológicos, Instituto Nacional de Antropología e Historia, Ciudad de México, México josuhe_lozada@inah.gob.mx (iD) $0000-0002-6203-2513$ 


\section{Introducción}

$\mathrm{E}$ n la interminable búsqueda del ser humano de la eternidad, dejar una marca en la roca es una de las soluciones más antiguas y efectivas que existen. Estudiando el arte rupestre se aprecia la complejidad cognitiva de las más antiguas sociedades, pero no solo de las culturas arcaicas, porque este arte se realizó de una manera constante y se transformó con el tiempo a la par de triunfos y derrotas, recordando sequías, guerras o celebraciones. ${ }^{1}$

El objetivo de este trabajo es presentar el registro del arte rupestre de Laguna Miramar que, visto desde un enfoque regional, contribuye a la comprensión de los estilos y las temporalidades del arte rupestre plasmado en los riscos situados alrededor de las lagunas de la Selva Lacandona. Estas manifestaciones artísticas son una fuente de información alternativa para identificar temas religiosos, temporalidades y estratos culturales que suelen pasar desapercibidos y rara vez se estudian en conjunto con las evidencias arqueológicas asociadas.

Para registrar las pinturas se fotografiaron a diferentes horas del día, se tomaron medidas directas y se hicieron bocetos con observaciones relativas a color (Munsell), forma y observaciones respecto al estado de conservación y otros detalles. En gabinete, con el programa DStrech se resaltaron los diseños menos visibles, lo que aumentó considerablemente la cantidad de diseños conocidos para el sitio. Por último, se obtuvieron archivos JPG de cada diseño con escala y se realizaron dibujos en conjunto de cada panel de pinturas.

Para los petrograbados se realizó un dibujo in situ a manera de croquis, observando la posición de cada petrograbado en el muro de roca — se realizaron observaciones a diferentes horas del día-. En seguida se fotografió a escala cada elemento, así como el panel en general. Se tomaron medidas de alto y ancho de cada elemento identificado, la distancia entre estos, la orientación y la inclinación general del conjunto. Los dibujos digitales se basaron en las fotografías y se corrigió la distorsión óptica con las medidas reales.

El estudio del arte rupestre en Chiapas inicia en el Planchón de las Figuras y las pinturas de Laguna Pethá por Teobert Maler en 1903. En los años cuarenta del siglo pasado, Heinrich Berlín escribió en sus notas algunas descripciones y comentarios sobre la presencia de gráfica rupestre zoque camino a Copainalá, en la región de la Depresión Central del estado de Chiapas (Lozada, 2017). Frans Blom, en esos mismos años, registra algunos petrograbados en el municipio de Chapultenango. ${ }^{2}$

Los grafitis plasmados sobre arquitectura monumental maya prehispánica, aunque no son arte rupestre propiamente, constituyen un acervo de almacenaje simbólico importante para el estudio del fenómeno rupestre. En Yaxchilán se registraron pinturas de manos rojas en el Templo 33 en 1891 (López de Llergo, 1925); Maler (1903) también aporta algunos comentarios respecto a estos grafitis, y Scherer y Golden (2012) igualmente registraron manos rojas en Budsiljá.

\footnotetext{
${ }^{1}$ Agradecemos a las personas e instituciones que hicieron posible esta investigación: a la Escuela Nacional de Antropología e Historia, alma mater de ambos autores y a la Universidad Estatal de Arizona; a los habitantes y autoridades del Ejido Emiliano Zapata y a la Comisión Nacional de Áreas Protegidas (CONANP) por facilitar el acceso a los sitios, y a quienes dictaminaron el artículo por sus invaluables observaciones y comentarios.

${ }^{2}$ Puede consultarse el archivo del museo Na Bolom en San Cristóbal de Las Casas, Chiapas.
} 
Por otro lado, la New World Archaeological Foundation registró desde 1953 en diversos trabajos algunos motivos rupestres que, tiempo después, Sophia Pincemin recopiló para la presentación de un proyecto de registro sistemático ante la Universidad de Ciencias y Artes de Chiapas para contar con un catálogo general y sistemático de dichas manifestaciones culturales. El resultado de esa investigación quedó fundamentado en la obra De manos y soles. Un estudio de la gráfica rupestre de Chiapas (Pincemin, 1999), que representa la fuente más importante a nivel de recopilación de datos en torno al estudio del arte rupestre en la entidad (Lozada, 2010).

En 1993 la Universidad Nacional Autónoma de México, a través del Instituto de Investigaciones Filológicas y el Centro de Estudios Mayas, publicó Un catálogo de frontera. Esculturas, petroglifos y pinturas de la región media del Grijalva, Chiapas (Navarrete, Lee y Silva, 1993), donde los autores resumen más de treinta años de investigaciones en la cuenca del Grijalva, con énfasis en los salvamentos arqueológicos de las presas La Angostura y Malpaso.

Las cédulas de registro que conserva la Sección de Arqueología del Instituto Nacional de Antropología e Historia (INAH) en Chiapas también son importantes para entender la presencia de arte rupestre en la entidad y conocer su distribución y tipología. En los últimos años se han registrado ocho sitios arqueológicos rupestres en la región de Ocozocoautla de este estado: la Sima del Copal (Acosta, 2004; Acosta y Méndez, 2006; Lozada, 2010), la Sima del Tigre, la Sima del Mujú, la cueva de La Cotorra, la cueva de Los Perros, la cueva La Encañada, el abrigo de Santa Marta y el abrigo Los Grifos (Acosta, 2008). Se reportan además otros sitios en el cañón del río La Venta (Acosta y García, 2005), El Naranjo (Acosta y Lozada, 2006) en el municipio de Berriozábal y 21 sitios con representaciones rupestres ubicados en el Cañón del Sumidero (Acosta et al., 2010).

Aunque el estado de Chiapas es rico en arte rupestre, la presencia de este en la literatura arqueológica aún es marginal. ${ }^{3}$ La Selva Lacandona, junto con la zona zoque, es donde más estudios se han realizado, aunque quedan grandes áreas aún sin explorar.

Los sitios rupestres que se han registrado hasta el momento en la selva de Chiapas se clasifican en cuatro tipos:

1. Riscos con arte rupestre situados en lagunas: Laguna Miramar (Folch, 2018), Laguna Pethá (Lozada, 2018) y Laguna Mensabak (Lozada, 2017).

2. Bloques de piedra esculpidos: Xoc (Ekholm-Miller, 1973), El Muñeco (Stein, 1979; Folch, 2018) y la Roca de Los Sacrificios (Palka, 2014).

3. Planchones de piedra caliza grabada: Planchón de Las Figuras (García, 1995).

4. Cuevas con arte rupestre: pueden tener pintura como la Cueva de Las Manos Rojas (Scherer y Golden, 2012), la Cueva Joloniel (Bassie, 2006) y la Cueva Yaleltsemen (Strecker, 2008), o pueden contar con petrograbados como la Cueva del Elefante (Folch, 2018) y la Cueva Max (Lozada, 2017).

Como se puede deducir de lo escrito anteriormente, se ha generado un fuerte impulso por estudiar el arte rupestre en las últimas dos décadas, el cual pretendemos continuar con el presente trabajo.

\footnotetext{
${ }^{3}$ Ver: Méndez (1998, 1999, 2010), Pincemin (1999), Céra (1977, 1980), Gussinyer (1976, 1980), Navarrete $(1959,1966,1992)$, Navarrete y Martínez (1961), Navarrete, Lee y Silva (1993), Strecker $(1987,2008)$ y Weber y Strecker (1977).
} 


\section{El área de la Selva Lacandona y Laguna Miramar}

Laguna Miramar se ubica en la parte sur de la Selva Lacandona, en la región geológica conocida como Montañas de Oriente (Müllerried, 1957), y forma parte de la cuenca del rio Jataté, que nace cerca de la ciudad de Ocosingo y corre en dirección sureste hasta juntarse con el río Santo Domingo para formar el río Lacantún, que más adelante se convierte en el Usumacinta.

La geomorfología de la Selva Lacandona consiste en su totalidad de rocas sedimentarias calizas de los periodos Cretácico, Terciario y Cuaternario (Ortega et al., 1992). Es una formación de origen tectónico que moldeó plegamientos, los cuales crearon una serie de valles que corren diagonalmente de noroeste a sureste. Colinda al sur con la Franja Transversal del Norte y los Cuchumatanes de Guatemala, al oriente con las planicies del Petén también de Guatemala, al norte con la planicie costera del Golfo de México y al poniente con Los Altos de Chiapas y la región de los Llanos de Comitán.

Laguna Miramar (ver Figura 1) se localiza en el valle de San Quintín, que tiene una extensión de 27200 hectáreas (González, 1995), limita al norte con el cerro Mono Blanco, al poniente con la Sierra de la Colmena, al sur con el Cordón Chaquistero y al oriente con la Sierra Caribe (Rivero, 1992, p. 15). La mitad oriental del Valle, incluida Laguna Miramar, se localiza dentro de la Reserva de la Biosfera Montes Azules. Al centro del valle se levanta el cerro Chuncerro o Chunwitz (también conocido como Chum-witz), que tiene forma de herradura y delimita la parte sur de Laguna Miramar, mientras la parte norte es delimitada por el cerro Mirador (también nombrado Pico Pelón o cerro Miramar). Cuenta con cuatro tipos de rocas sedimentarias: continentales en la mayor parte del valle, producto de la erosión hídrica de los ríos Jataté y Perlas; de origen marino en la parte alta y media de las montañas, y de origen mixto en la parte baja de los cerros (Ortega et al., 1992). Adicionalmente se reportan manantiales de aguas azufrosas en algunos puntos (Blom y Duby, 1957).

Figura 1. Sitios arqueológicos de Laguna Miramar, Ocosingo, Chiapas

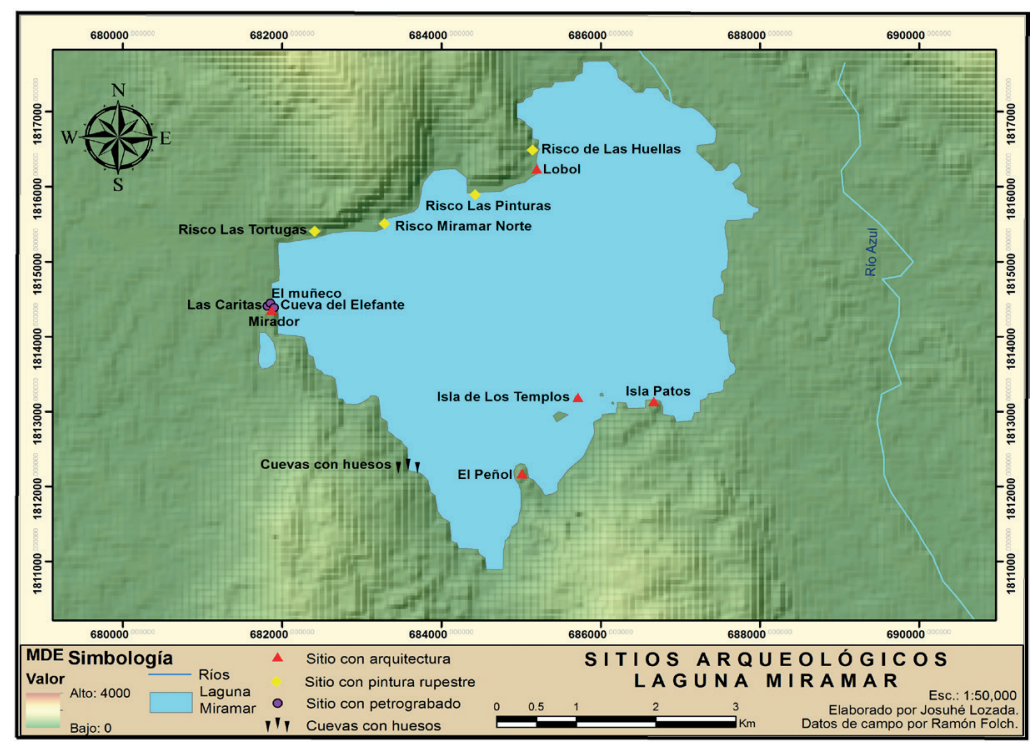

Fuente: elaborado por Josuhé Lozada Toledo. 
Laguna Miramar tiene forma de diamante, una extensión aproximada de 16 kilómetros cuadrados y seis kilómetros de diámetro. Dentro del cuerpo de agua hay varias islas, las más grandes se ubican en la ribera sur. Sus aguas son cálidas, color turquesa y cristalinas; se puede ver a más de diez metros de profundidad fácilmente. La profundidad del lago es desconocida, aunque por analogía con Laguna Lachúa, en Guatemala, cuya formación y características son casi idénticas, se pueden esperar profundidades que superan los 200 metros (Machorro, 2005; Unión Internacional para la Conservación de la Naturaleza e Instituto Nacional de Bosques, 1999). La formación del lago se debe a un Polje (Erben, 1955), es decir, una gran extensión de tierra que colapsó a causa de la erosión hídrica subterránea, por lo cual la mayor parte de las riberas del lago no tiene costa y se crean riscos que caen directamente al agua.

\section{Estudios previos en Laguna Miramar}

Laguna Miramar fue visitada por los conquistadores españoles en 1530 durante una entrada a la selva al mando de Alonso Dávila. La segunda entrada española a dicha laguna se llevó a cabo en 1559 bajo Pedro Ramírez de Quiñones, y la tercera y última expedición en 1586 al mando de Juan de Morales Villavicencio. Estas tres entradas pioneras a Laguna Miramar significaron guerra contra el grupo chollacandón que ahí habitaba y su obligado abandono del sitio (Blom, 1956; De Vos, 1996).

El área fue redescubierta en 1876 por Juan Ballinas, y el lago en 1880 por los madereros de la compañía Bulnes. La laguna fue explorada científicamente en 1934 por Jacques y Georgette Soustelle (etnólogos), y en 1936 por David W. Amram (geógrafo). La primera investigación formal del lago la realizó Frans Blom en 1950, y se registró otra expedición en 1955 a cargo del Centro de Investigaciones Antropológicas de México (CIAM) bajo la dirección del etnólogo Juan D. Leonard. En los años setenta fue explorada por Guillermo Stein, quien solía llevar turistas y dio a conocer el grabado del Muñeco (Folch, 2018). Los primeros que reportaron haber visto pinturas rupestres fueron Arturo Bayona y Adriana de Castro (1994, p. 104) en 1979, aunque publicaron sus resultados en 1994.

En 1985, la arqueóloga Sonia E. Rivero organizó las únicas investigaciones en la zona a cargo del INAH. Bajo el Proyecto Miramar se realizaron excavaciones en las islas al sur del lago y se registró la pintura rupestre con dibujos muy esquemáticos y descripciones breves (Rivero, 1985, 1988, 1989, 1992).

Tras el movimiento zapatista de 1994, las pinturas rupestres de Miramar han sido visitadas por Carlos Tello Díaz (2004) en 2001 y por Ramón Folch González (2018) entre 2013 y 2019. Este último autor realizó un registro sistemático de las pinturas en el año 2017 con ayuda de dos estudiantes de la Escuela Nacional de Antropología e Historia (ENAH). Las pinturas de Laguna Miramar han sido mencionadas por Pincemin (1999) y por Künne y Strecker (2008) al citar el trabajo de Rivero (1992).

Hasta la fecha se conocen cuatro riscos con pintura rupestre, todos ubicados en la ribera norte. En la ribera oeste se encuentra la zona de cuevas con dos riscos y una cueva con petrograbados (ver Figura 1). En un primer momento se tratarán las pinturas y posteriormente se describirán los petrograbados.

\section{Los riscos con pintura rupestre de Laguna Miramar}

El Risco de las Pinturas se localiza al norte de Laguna Miramar; ubicado junto al agua, se accede únicamente en cayuco o lancha tras remar aproximadamente 45 minutos desde el campamento de 
Emiliano Zapata con un guía de la comunidad. El risco tiene catorce metros de largo por ocho de altura, y la mayoría de los motivos se localizan a una altura que va de uno a tres metros.

Figura 2. Dibujo de las pinturas del Risco de las Pinturas

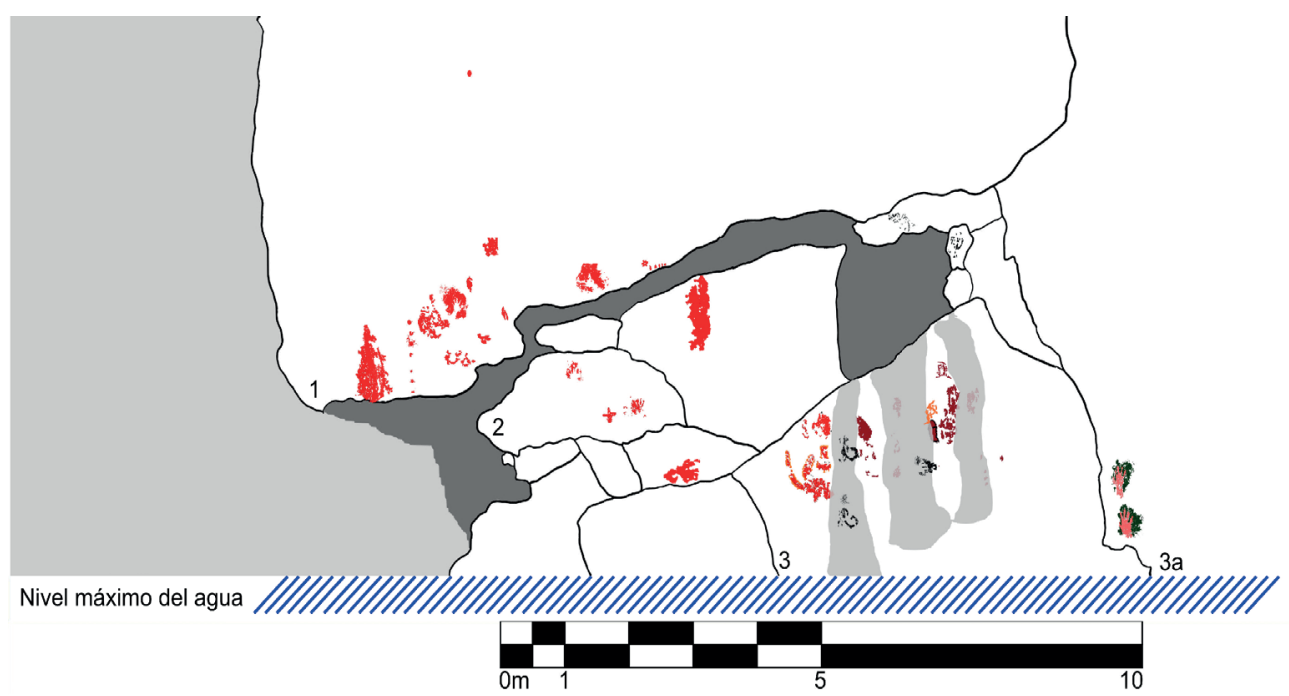

Risco de "Las Pinturas", Laguna Miramar, Chiapas, México

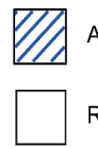

Agua de la laguna

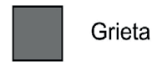

Risco de roca caliza

Zona cubierta por

escurrimientos de sales

Fuente: elaborado por Ramón Folch González, 2017.

Es un risco de piedra caliza de color blanco con manchas negras producidas por la presencia de microflora e intemperismo. Se subdivide en varios bloques que forman paneles con distintos momentos de ejecución, en este caso se pueden contar cuatro (1, 2, 3 y 3a), aunque la vegetación podría esconder otras pinturas. Los colores de las pinturas son negro, rojo ocre, naranja, rojo oscuro, verde oscuro y rojo claro (ver figuras 2 y 3). El agua de lluvia no cae directamente sobre las pinturas, aunque sí han afectado mucho su conservación los escurrimientos de agua, en particular en el Panel 3; los diseños han sido borrados por las sales y carbonatos que precipitan de la roca caliza, o en algunos casos se localizan debajo de la capa de carbonatos.

Las pinturas del risco se pueden dividir en dos tipos: amorfas y diseños. Los manchones amorfos son aquellas manifestaciones sin forma aparente o que han sido borradas hasta ser indistinguibles. Los diseños son aquellos dibujos que se aprecian claramente y pueden ser geométricos, antropomorfos, zoomorfos, manos y fitomorfos.

Estratigráficamente se reconocen tres momentos de ejecución, los que se describen a continuación y que serán discutidos más adelante (ver Figura 3). 
Figura 3. Estratigrafía de los pigmentos del Risco de Las Pinturas señalando cada momento pictórico

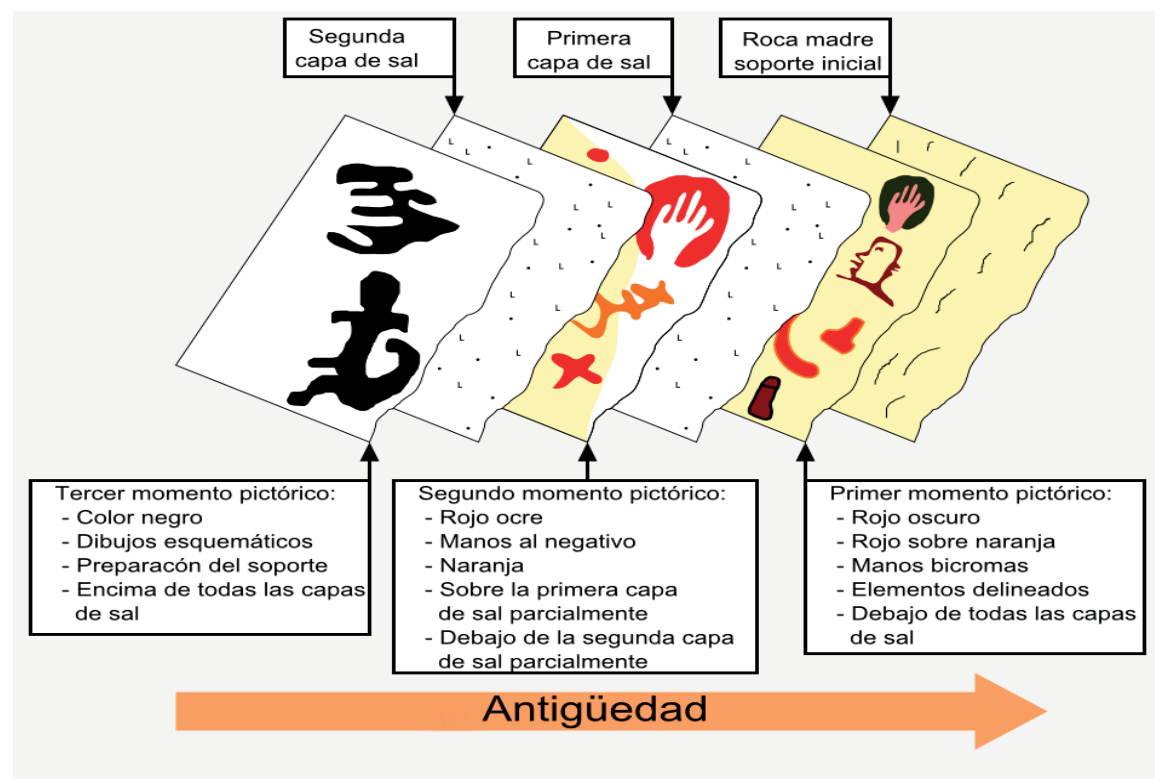

Fuente: elaborado por Ramón Folch González.

El primer momento de producción rupestre refiere al Panel 3, que fue pintado directamente sobre el soporte rocoso. En sus diseños, más elaborados que los posteriores, se utilizan por lo general dos colores, y la línea se pinta con pincel o alguna herramienta que permite trazar líneas regulares. Los colores utilizados son rojo oscuro (7.5R 3/4), rojo delineado de negro (5R $3 / 6$ y $5 \mathrm{R} 4 / 1$ ) y rojo delineado de naranja (7.5 R 4/4 y 5YR 5/6). La mayoría de los motivos de este momento no se logran identificar, por lo que se registran como manchones; los diseños identificados son una cabeza humana, un manchón con forma de pierna o falo y un elemento semicircular. Se propone incluir en este momento las dos manos bicromas pintadas del Panel 3a pintadas en verde oscuro y rosa (7.5R 7/3 y 1 GLEY 4/5 G), pigmentos que no están presentes en los momentos más tardíos ni en otros sitios conocidos de la región.

Encima de la primera capa de sales formada sobre el primer momento de producción, se plasman imágenes en un segundo momento pictórico (Folch, 2018, p. 112) a manera de una capa pictórica sobrepuesta; son diseños notablemente diferentes: manos al negativo, puntos, un diseño geométrico circular con líneas que le rodean a manera de un sol, una cruz, un motivo zoomorfo y un fitomorfo, todos de color rojo ocre (7.5 R 5/6), con excepción del sol que es anaranjado (2.5 YR 5/8), y distribuidos en el Panel 1 y el Panel 2. Estos diseños se realizaron sin uso de pincel, con la técnica de aspersión, que consiste en escupir la pintura directamente con la boca o mediante algún artefacto hueco. El último momento pictórico se encuentra únicamente sobre el Panel 3, y específicamente sobre la última capa de sal; quizás los productores del arte rupestre buscaron la parte más blanca del risco por alguna razón estética o simbólica. Son tres diseños, dos monos y un par de manos, todos de color negro (2.5 Y 2.5/1) y esquemáticos. Una particularidad de este momento es que se preparó el soporte antes de pintar los diseños, pues se observa que se golpeó un pedazo de pared rocosa para poder pintar la cabeza de uno de los monos (ver Figura 4). 
Figura 4. Detalle del diseño de mono color negro del periodo histórico

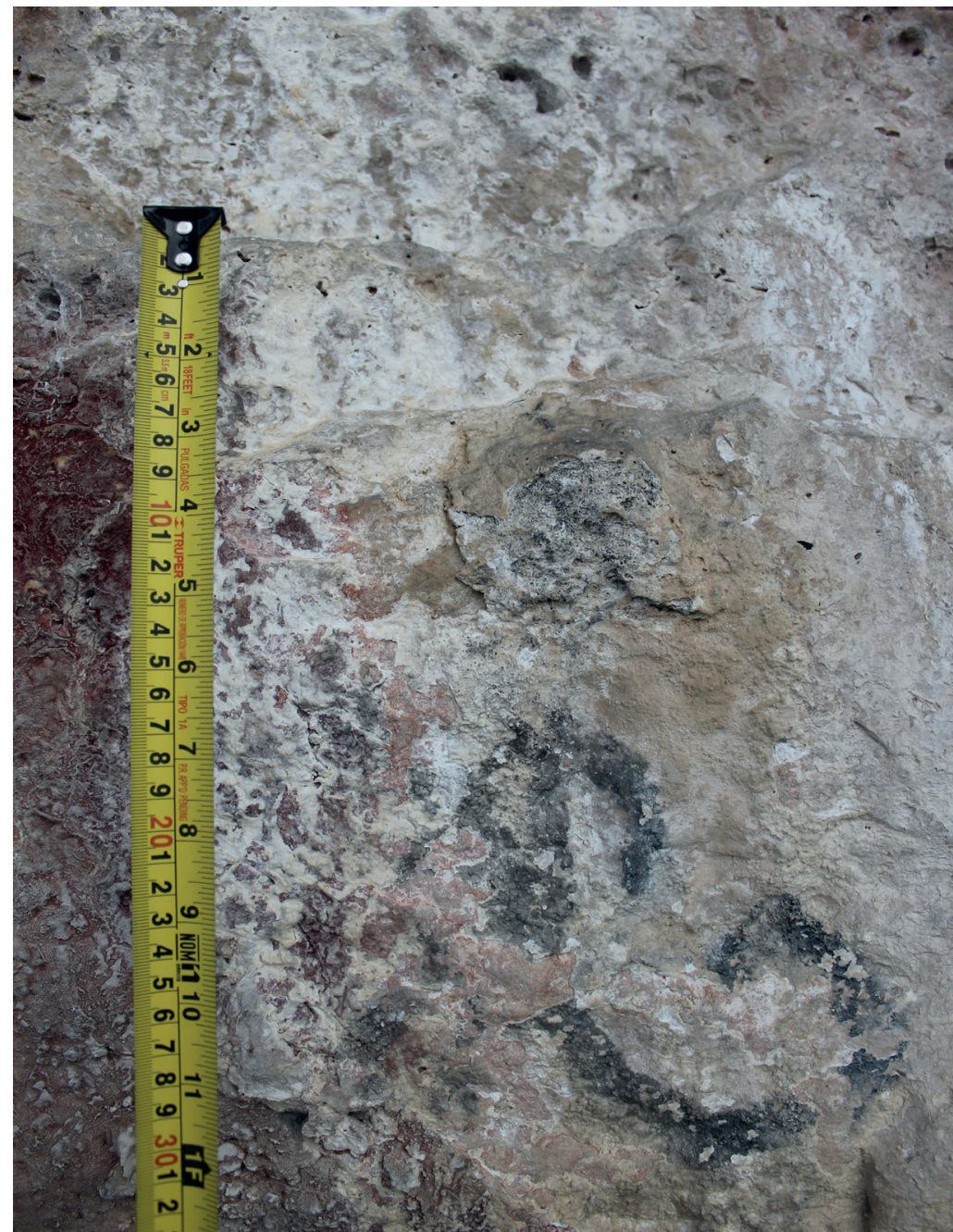

Fuente: foto de Ramón Folch González. Nótese la superficie golpeada en el sitio de la cabeza.

El Risco de las Tortugas es de grandes dimensiones y a él se accede también por medio de cayuco o lancha. Es probable que este sitio haya tenido importancia debido a su ubicación en la base del cerro Mirador o Miramar, a la entrada de una gran grieta conocida localmente como Cueva de las Tortugas.

En la entrada de la cueva se desprende un enorme risco blanco que presenta motivos geométricos muy erosionados pintados en rojo. Se trata de cinco puntos rojos divididos en dos grupos, uno con tres puntos que forman un triángulo y el segundo con dos. El color rojo y la composición esquemática de los puntos pueden relacionar este risco con el segundo momento pictórico del Risco de las Pinturas.

El Risco Miramar Norte es de menores dimensiones que los dos anteriores y está a medio camino entre aquellos. Es un pequeño risco que tuvo algunos diseños, pero han sido erosionados por el intemperismo. Toda la pintura en el Risco 3 es rojo ocre, por lo que se relaciona con el segundo momento pictórico. 
El Risco de las Huellas se relaciona con un abrigo rocoso en el cual hay restos de hollín, resina quemada y desprendimiento deliberado de estalactitas en la antigüedad. A la entrada del abrigo se tallaron bajo el agua cuatro huellas humanas, y sobre la pared algunos metros al este se pintó una mano de color rojo. De nuevo el color rojo se asocia al segundo momento pictórico (ver Figura 5).

Figura 5. Vista y detalles del Risco de las Huellas, al noreste de Laguna Miramar

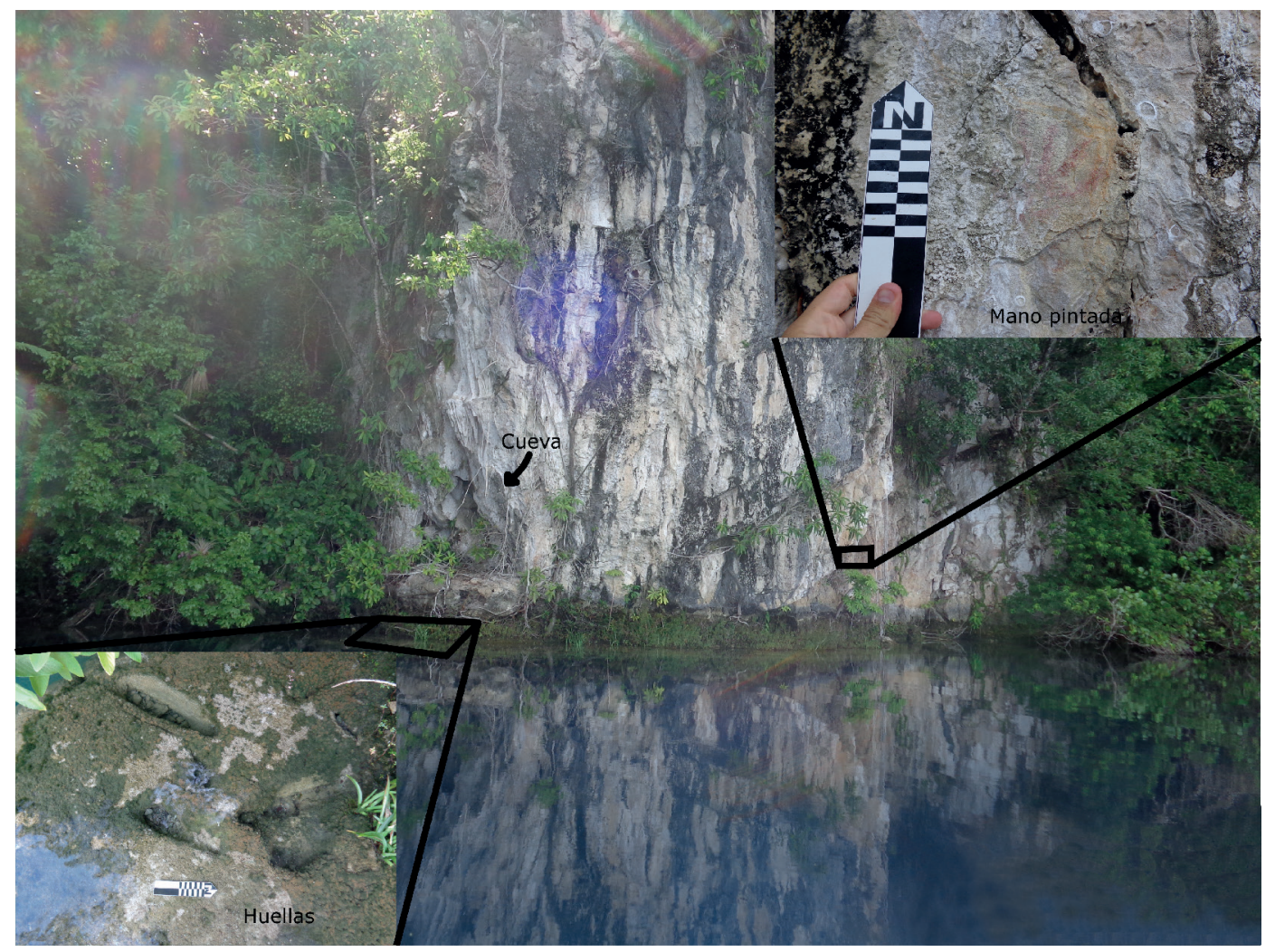

Fuente: foto de Ramón Folch González.

A continuación se discutirá sobre los estilos representados en el arte rupestre de Laguna Miramar a partir de un análisis comparativo con otros sitios rupestres de la región de la Selva Lacandona. Se profundizará sobre las temporalidades de ejecución de las pinturas y petrograbados, que nos remiten desde el horizonte Preclásico Tardío hasta los tiempos históricos.

\section{Arte rupestre de estilo Preclásico}

En comparación con las pinturas de Mensabak, que han sido sistemáticamente estudiadas (Lozada, 2017; Palka y Lozada, 2018), hemos podido detectar una temporalidad idéntica. En ambos sitios se reportan tres momentos pictóricos: uno en el Preclásico Tardío, otro en el Posclásico y el más reciente en tiempos históricos (siglos XVII-XIX). 
Durante el Preclásico Tardío se realizaron las pinturas con mayor diversidad de colores y formas, con utensilios más finos como pinceles. Los colores utilizados en este periodo son: el rojo oscuro, cuyas tonalidades se aprecian en la mayoría de las imágenes más antiguas en el Risco Tsibaná en Laguna Mensabak y en Laguna Miramar, el rojo oscuro delineado en negro, el rojo oscuro delineado en anaranjado y manos realizadas con técnica mixta en colores verde oscuro y rosa.

Los diseños de este periodo comprenden diseños complejos y asociados a la élite gobernante. En el Risco Tsibaná en Laguna Mensabak hay figuras humanas de gran tamaño, cuya posición del dorso visto de frente, y rostro y pies vistos de perfil, recuerdan un estilo de características olmecas (Lozada, 2017).

Laguna Miramar cuenta con una cabeza también de rasgos "olmecoides" (ver Figura 6), acompañada de manos de técnica mixta nunca antes reportadas en el área y otras figuras de gran formato, así como también se aprecian diseños complejos cubiertos por la precipitación de sales.

Figura 6. Algunos de los diseños asociados al momento Preclásico en el Risco de las Pinturas
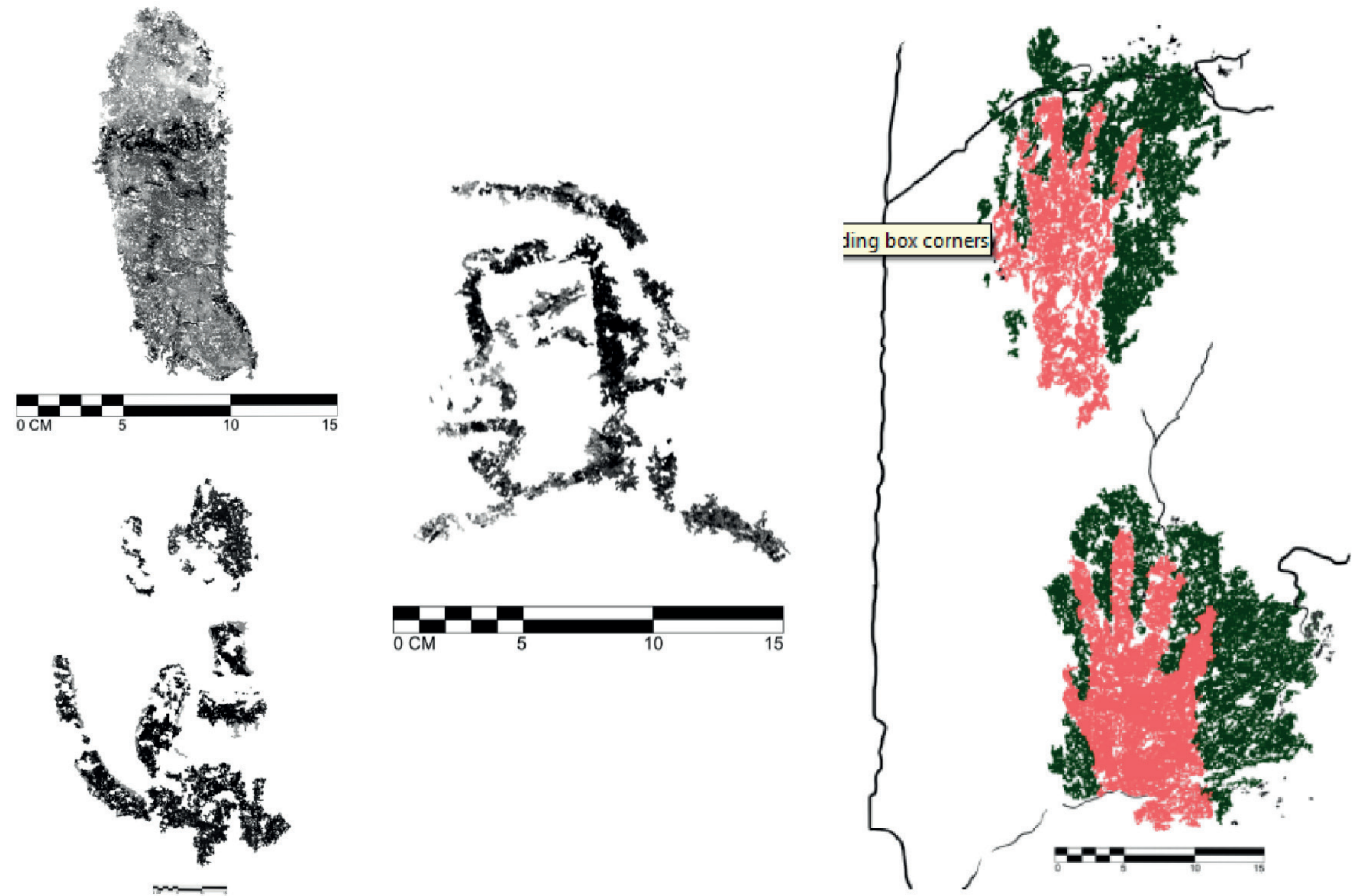

Fuente: elaborado por Ramón Folch González.

Todas estas representaciones gráficas, proponemos, pertenecen al periodo Preclásico Tardío (300 a.C. a 200 d.C.); utilizando la estratigrafía de las pinturas (ver Figura 3), se puede valorar que estos motivos se encuentran debajo de los dos estratos salinos y de las pinturas más recientes. 


\section{Arte rupestre de estilo Clásico Tardío-Posclásico}

Estratigráficamente hay una clara división entre los motivos más antiguos, cubiertos por una primera capa de sal, y el segundo momento pictórico que proponemos fechar hacia el Clásico Terminal (800 d.C. a 900 d.C.) y todo el periodo Posclásico (900 d.C. a la llegada de los españoles). La casi totalidad de las pinturas fueron plasmadas utilizando un pigmento rojo ocre y en una menor parte en naranja, con diseños más sencillos que consisten en: manos al negativo y al positivo, cruces, puntos, diseños astronómicos y representaciones de animales.

Tanto en Laguna Miramar como en Laguna Mensabak y Laguna Pethá, el color rojo ocre (7.5 R 5/6) se encuentra en la mayoría de las pinturas, por lo que se puede considerar una característica diagnóstica del arte rupestre del periodo Posclásico.

En Laguna Miramar las pinturas de este periodo ocupan todos los paneles del Risco de las Pinturas, el de las Tortugas, el de las Huellas y el Risco Miramar Norte. La pintura roja está también presente en zonas como Yaxchilán (López de Llergo, 1925, p. 364), Budsiljá (Scherer y Golden, 2012, pp. 48-49) y la Cueva de las Manos Rojas (Scherer y Golden, 2012, pp. 54-58), por lo que parece haber sido una época de mucho peregrinaje por las ciudades en ruinas de la Selva Lacandona. Refiriéndonos a peregrinajes, uno de los diseños que más llama la atención del Risco de las Pinturas es la figura de un animal dibujado al negativo con la técnica de sopleteo, del cual solo se conocen ejemplos semejantes en Oxkutzcab, Yucatán (Strecker, 1982), El Salvador ${ }^{4}$ y la zona zoque al oeste de Chiapas (Lozada, 2010). Lo anterior fortalece la idea de amplios recorridos rituales durante el Posclásico (Palka, 2014).

\section{Arte rupestre de estilo Histórico}

El periodo histórico es el tercer momento pictórico que se propone para las representaciones rupestres de Laguna Miramar. Se plantea su ubicación hacia los siglos XVIII y XIX, relacionado con lacandones de habla maya-yucateco, ancestros de los lacandones actuales. Estos motivos corresponden con aquellos que los lacandones plasmaron en sus jícaras y su vestimenta en la primera mitad del siglo XX, por lo que se aprecia una continuidad en la tradición pictórica maya.

Se cuenta únicamente con tres diseños plasmados encima de la segunda capa de sal que cubrió los motivos más antiguos. Todas las imágenes de este periodo se pintaron en negro, tanto en Laguna Miramar como en Laguna Mensabak y Laguna Pethá, son diseños elaborados con trazos muy simples (Lozada, 2017). En Laguna Miramar se registran dos monos y un diseño compuesto de dos manos pintadas al negativo (cuya pintura queda entre las dos manos), siendo probable que el hecho de estar plasmadas deliberadamente sobre la segunda capa de sal indique que los lacandones históricos que las representaron buscaron un fondo blanco para el diseño, asociado a pinturas más antiguas.

\footnotetext{
${ }^{4}$ Comunicación personal de Félix Lerma, 2019.
} 


\section{Petrograbados de Laguna Miramar}

En la zona de cuevas de Laguna Miramar existen muchas evidencias arqueológicas de los habitantes del pasado, entre ellas petrograbados dentro y fuera de cuevas. El relieve del Muñeco es el más sobresaliente de estos.

Además del Muñeco, se encuentra un panel de grabados al que denominamos Caritas en el mismo risco donde se ubica el Muñeco, pero alrededor de 80 metros al oeste. Al sur del Muñeco se localiza la Cueva del Elefante, que es el paso obligado para subir al cerro Mirador, el cual cuenta con una plataforma y terrazas; en esta cueva hay una estalagmita grabada con un rostro humano. Estos son los tres ejemplos conocidos hasta ahora en el lugar que no ha podido ser explorado intensivamente.

El Muñeco fue descubierto por chicleros en el año 1970 y dado a conocer al mundo por Stein (1979); posteriormente fue descrito por Sonia Rivero (1992) y Arturo Bayona y Adriana de Castro (1994), y fue igualmente visitado entre 2013 y 2019 por Folch (2018). El análisis de Stein sobre el bajorrelieve es consistente, por lo que nuestras interpretaciones se basan en su mismo trabajo.

Stein en 1979 lo describe de la siguiente manera:

Ya en 1972 me llevaron indios de San Quintín al acantilado de piedras de la [Cueva del Mono]. Se supone que fue descubierto casualmente dos años antes por un chiclero. Se encuentra ubicado en una pared escarpada de la roca que tiene una altura de unos 25 metros y que se extiende hasta el lago. Unos cuatro metros a la derecha del relieve en la cara de la roca en apertura estrecha (alrededor de 0.70 metros de ancho) que recibe su nombre al parecer de la figura tallada de la pared de roca como son también llamadas [mono] las esculturas antiguas por los nativos.

El relieve está cerca de 15 metros de altura y tiene un metro de altura. Representa una figura humana sentada en un banco. A pesar de la fuerte erosión algunos detalles se conservan, como la mano izquierda, cuidadosamente esculpida. La cabeza parece estar coronada por un casco (Stein, 1979: $61-62) .^{5}$

Podemos agregar a lo dicho por Stein que el relieve mira al norte y que la inclinación de la pared rocosa en la que se encuentra hace que el relieve mire hacia abajo, al espectador, animando al objeto. Tiene en el lado izquierdo del rostro restos de lo que asemeja un bigote.

Navarrete (1972) relaciona estas figuras con la fase Hato de Izapa hacia el Preclásico Tardío, entre los años 0 y 100 de nuestra era. Es interesante notar que todos los ejemplos de estas figuras se encuentran en escultura y cerámica, únicamente uno proveniente de excavaciones arqueológicas, el cual pudo ser fechado por asociación a vasijas de la fase antes mencionada.

El Muñeco presenta varias particularidades que lo hacen único; en primer lugar, su tamaño. Todas las representaciones citadas por Navarrete (1972) son de pequeño formato, pero esta mide un poco más de un metro de alto y algo menos de un metro de ancho. Otra peculiaridad es que se trata de un bajorrelieve, en tanto que los demás ejemplos son esculturas en piedra pulida o en barro.

${ }^{5}$ Traducción del alemán por R. Folch y J. Welbers. 
El nicho en que se encuentra la figura está redondeado en su parte alta, lo cual podría representar una cueva; además está junto a la entrada a una cueva en la que se han encontrado materiales arqueológicos como cerámica y huesos cuya temporalidad se desconoce (Bayona y De Castro, 1994, pp. 219-220). Esta posible representación de un personaje de alto rango saliendo de una cueva podría compararse a los altares de La Venta en Tabasco o a los relieves de Chalcatzingo, Morelos, que también son del Preclásico. El elemento del personaje asociado a una cueva (oquedad) puede vincularse con el inframundo.

\section{Las Caritas}

Al oeste del petrograbado del Muñeco, en la misma pared rocosa, se encuentra en un talud natural de roca una decena de grabados que forman rostros esquemáticos, conocidos en la literatura arqueológica como "Caritas" (ver Figura 7).

El registro de este panel se realizó en enero de 2017 durante una visita a la Laguna de Miramar acompañado por dos estudiantes de la ENAH, además del guía asignado por la comunidad Emiliano Zapata.

Figura 7. Dibujo del panel de Caritas en la zona de cuevas

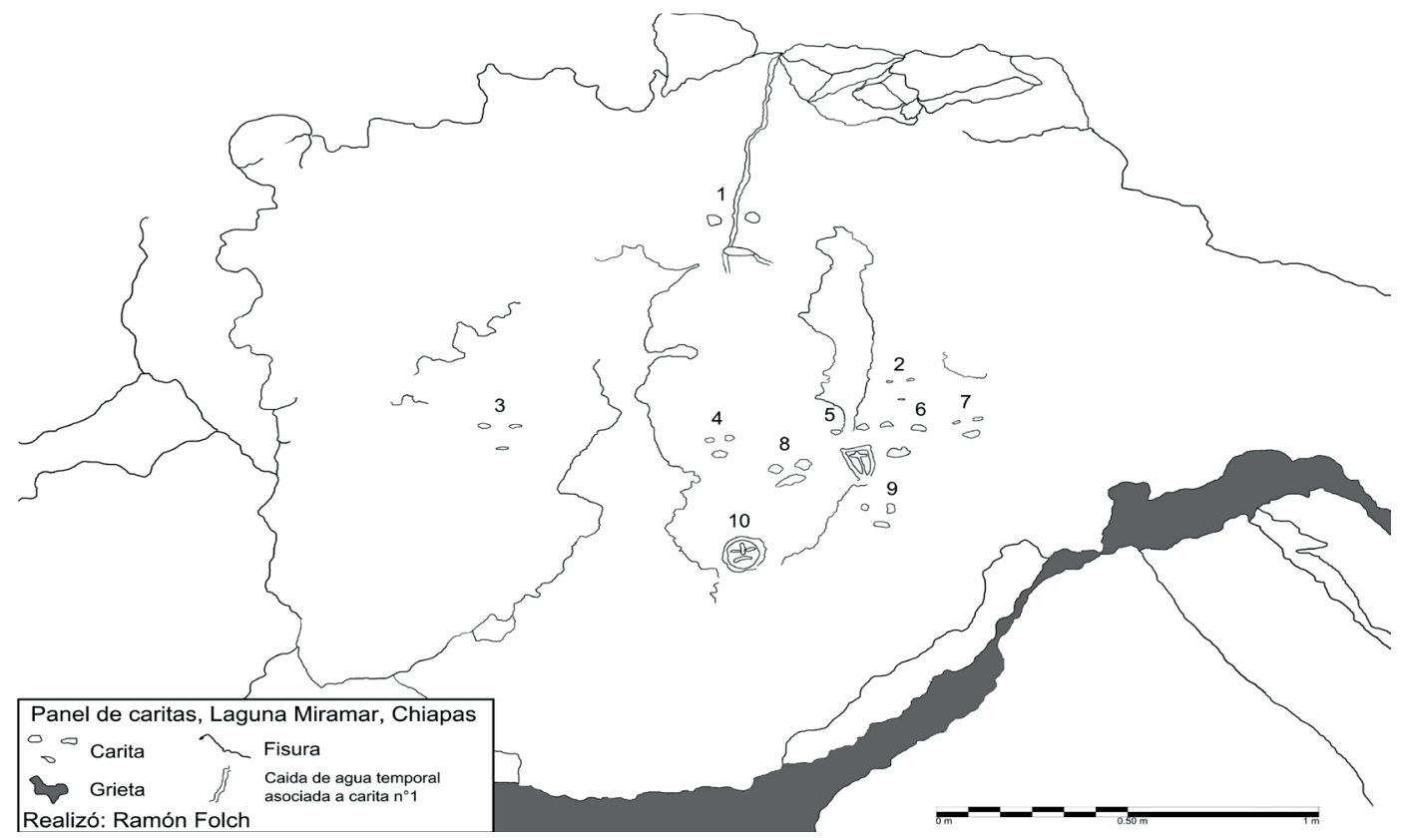

Fuente: elaborado por Ramón Folch González. Nótese la corriente de agua que "alimenta" a la Carita número 1.

Entre las de este panel, destacan la Carita número 1 y la 10, así como un diseño geométrico en forma de cruz realizado de tal manera que se reutilizó la boca de la Carita número 5 . El resto consiste en rostros formados por dos puntos que representan los ojos y una línea horizontal que representa la boca; la mayoría se encuentran agrupadas a la misma altura, con excepción de la 1 y la 10. 
La Carita 1 se ubica a lo alto del panel sobre una canaleta natural resultado del escurrimiento de agua; esta canaleta vierte el agua directamente en la Carita, lo cual hace que la corriente de agua se divida en dos y fluya por la comisura de la boca, dando la impresión de estar bebiendo. Lo anterior fue comprobado en campo vertiendo agua en la canaleta. Es la primera vez que se registran estas interacciones en petrograbados del área maya de este tipo. El dar de beber a esta representación la anima, convirtiéndola en una persona con la que se interactúa posiblemente para la petición de lluvia o de buenas cosechas, para solicitarle permiso para entrar a las cuevas o para algún otro requerimiento de carácter mágico-religioso. A la Carita 10 le representaron la nariz y la cabeza, y se localiza en la parte más baja del panel.

En Mesoamérica, las Caritas son algo común que se asocia con el culto al agua y con lugares de paso, y son localizadas en cuevas, riscos, cimas de montañas y ojos de agua. Tentativamente han sido fechadas hacia el Clásico Tardío y el periodo Posclásico; sin embargo, solo en algunos casos han sido fechadas por asociación con materiales cerámicos, por lo que es difícil asignarles una temporalidad.

La Carita 5 debe analizarse por separado dado que sobre ella se grabó una cruz dentro de un triángulo invertido, lo cual probablemente fue realizado mucho tiempo después por algún cristiano. Esta alteración pudo haber sido realizada durante alguna de las entradas de españoles a Laguna de Miramar, entre 1530 y 1585, aunque también existe la posibilidad de que dicha afectación fuera realizada por madereros o chicleros durante finales del siglo XIX o principios del XX, pues en repetidas ocasiones estos fueron responsables de deliberadas destrucciones a vestigios arqueológicos, como sucedió en Yaxchilán, y en la misma Laguna Miramar a una escultura en la Isla de los Templos en la década de los años sesenta (Schmidt, 1979). Dichos grupos solían asociar las esculturas antiguas con el diablo, por lo que cuando veían un muro con rostros era común que grabaran una cruz encima.

\section{La Cueva del Elefante}

Esta cueva está localizada a unos 80 metros al sur del relieve del Muñeco, en el camino para subir al Mirador. Aunque se denomina cueva, este lugar es un abrigo rocoso que recibe su nombre debido a una estalactita en forma de pata de paquidermo. A la mitad del abrigo se ubica una gran estalagmita sobre la que se talló un rostro aprovechando una protuberancia en forma de cabeza; los ojos y la boca están representados por círculos y una fisura asemeja el cabello (ver Figura 8).

Durante la expedición del CIAM, el arqueólogo Frederick Peterson realizó excavaciones al pie de este grabado, encontrando varios entierros, uno de ellos en cista con cerámicas asociadas del Clásico Temprano (Folch, 2018, p. 54).

Estos rostros en cueva se han reportado en la Cueva de los Andasolos (Navarrete y Martínez, 1961), en la cueva junto al Lago Bolonchac (Maler, 1903), en varias cuevas del Petén (Siffre, 1993), en Alta de la Paz (Woodfill, 2014) y en la Cueva del Rayo, en los alrededores de Comitán. ${ }^{6}$

En la base de la estalagmita se aprecia una Carita compuesta de tres oquedades circulares de carácter esquemático que corresponden a los ojos y la boca, lo que personifica la formación geológica, animándola. Las dimensiones de dicha Carita son de quince por nueve centímetros.

${ }^{6}$ En archivo del museo Na Bolom en San Cristóbal de Las Casas, Chiapas. 
Figura 8. Estalagmita grabada en la Cueva del Elefante

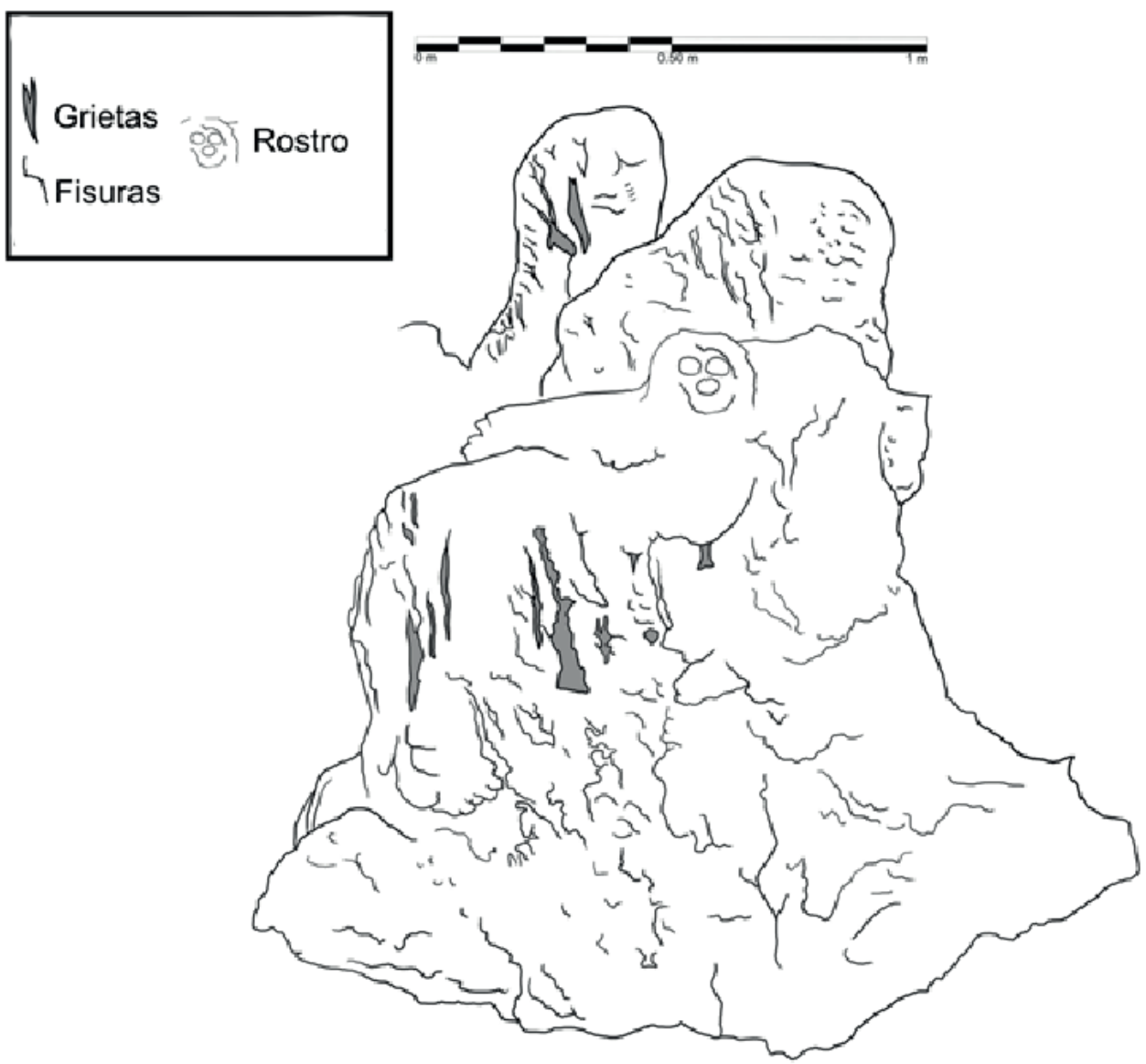

Fuente: elaborado por Ramón Folch González.

Este tipo de representaciones han sido interpretadas como guardianes de las cuevas, ayudantes del dios del agua o como la personificación de la cueva en sí. Debido a la presencia de entierros en esta cueva asociados directamente al espeleotema, podrían sugerir un culto a los ancestros, y considerase la cueva como una entrada al inframundo. Sumado a esto, es notorio el uso de estas cuevas como osarios desde el Clásico Tardío y el Posclásico hasta los tiempos históricos.

\section{Comentarios finales}

El estudio del arte rupestre de Laguna Miramar y su diversidad de motivos permite profundizar en el tema de los estilos y de las temporalidades en las representaciones rupestres de la selva tropical de Chiapas.

En los análisis comparativos con el arte rupestre de otras lagunas de la región nos hemos percatado de patrones culturales de los antiguos mayas, quienes eligieron los cuerpos de agua como lugares significativos para plasmar su sistema de almacenaje simbólico a partir de los motivos pintados o grabados sobre roca. 
Los riscos y cuevas alrededor de este cuerpo lacustre fueron el soporte de un sistema de creencias en cada momento de ocupación.

Desde el periodo Preclásico Tardío el arte rupestre fue importante en Laguna Miramar, en el que se enfatiza la importancia del cuerpo humano y de la persona humana, así como de las élites. Hacia el Clásico Tardío y Posclásico las temáticas rupestres se complejizan y multiplican, cobran importancia las "caritas" o rostros, las manos humanas y las figuras geométricas, mientras que en el periodo Histórico las figuras zoomorfas de monos y las manos humanas adquieren relevancia.

El estudio del arte rupestre ofrece una mirada sobre la complejidad del culto al lago y al paisaje. Miramar posiblemente fue destino de importantes peregrinaciones desde el periodo Preclásico; el grabado del Muñeco es un ejemplo de grupos lejanos que dejaron una huella importante en el sitio. Se cuenta con más ejemplos de visitas lejanas, como el caso de una vasija zoque localizada en una cueva cercana a Miramar y de esculturas de Toniná (Folch, 2018).

El arte rupestre de Laguna Miramar nos permite observar el culto popular en las épocas tardías cuando las élites parecen haber colapsado. Se pueden ver representaciones más sencillas como evidencia de una religiosidad popular que suele ser difícil de visualizar en el registro arqueológico.

\section{Referencias}

Acosta, G. (2004). Las pinturas de la Sima del Copal, Ocozocoautla, Chiapas [ponencia]. Simposio Prehistoria y Poblamiento de México, XXVII Mesa Redonda de la Sociedad Mexicana de Antropología e Historia, Xalapa, México.

Acosta, G. (2008). La cueva de Santa Marta y los cazadores-recolectores del Pleistoceno final-Holoceno temprano en las regiones tropicales de México [tesis de doctorado, Instituto de Investigaciones Antropológicas-Universidad Nacional Autónoma de México].

Acosta, G., y García, A. (2005, 15-18 de noviembre). Escalada prehispánica y actual: técnicas para el registro y protección de sitios rupestre en Chiapas [ponencia]. ler Simposio Nacional sobre Representaciones Rupestres. Instituto de Investigaciones Antropológicas-Universidad Nacional Autónoma de México.

Acosta, G., y Lozada, J. (2006, 6-9 de septiembre). El petrograbado del Naranjo: Una contribución metodológica y cronológica del acervo rupestre zoque [ponencia]. VII Coloquio Guatemalteco de Arte Rupestre. Universidad de San Carlos de Guatemala.

Acosta, G., Méndez, E., Ulloa, X., García, V., y Ozuna, I. (2010). Catálogo de sitios y elementos rupestres. En G. Acosta y E. Méndez (coords.), Proyecto Agricultura Temprana en el Área Norte de la Depresión Central. primera fase: recorrido de superficie área E15C68 - E15C69. Consejo de Arqueología-Instituto Nacional de Antropología e Historia.

Acosta, G., y Méndez, E. (2006). Representaciones rupestres en la región de Ocozocoautla”. En D. Aramori et al. (coords.), Presencia zoque. Una aproximación multidisciplinaria (pp. 307-321). Universidad de Ciencias y Artes de Chiapas; Consejo de Ciencia y Tecnología del Estado de Chiapas; Universidad Autónoma de Chiapas; Universidad Nacional Autónoma de México.

Bassie, Karen (2006). El proyecto de la Cueva de Jolja' (O. Chinchilla, trad.). FAMSI.

Bayona, A., y De Castro, A. (1994). Laguna Miramar: vida y experiencias. Inquietudes, Ediciones y Publicidad.

Blom, F. (1956). La gran laguna de los lacandones. Tlatoani, 10, 4-9.

Blom, F., y Duby, G. (1957). La Selva Lacandona: Andanzas arqueológicas. Editorial Cultura.

Céra, C. (1977). Peintures Rupestres Préhispaniques au Méxique. Mémoire de Maitrise; U.E.R. d'Histoire.

Céra, C. (1980). Hallazgo de pinturas rupestres en la cueva de la Chepa, Chiapas. Bulletin de la Mission Archeologique et Ethnologique Francaise au Mexique, 2, 7-13.

De Vos, J. (1996). La pazde Dios y del rey: La conquista de la Selva Lacandona (1525-1821). Fondo de Cultura Economica.

Ekholm-Miller, S. (1973). The Olmec rock carving at Xoc, Chiapas, Mexico (Papers of the New World Archaeological Foundation, 32). Brigham Young University. 
Erben, H. K. (1955). Informe preliminar. Geología (Expedición C.I.A.M. 1955). En Informe preliminar de la expedición a la Selva Lacandona oganizada por el Centro de Investigaciones Antropológicas de México. Centro de Investigaciones Antropológicas de México.

Folch, R. (2018). Historia y registro arqueológico de Laguna Miramar, Ocosingo, Chiapas [tesis de licenciatura, Instituto Nacional de Antropología e Historia; Escuela Nacional de Antropología e Historia.

García, R. (1995). Cuatroestudios sobreel Planchónde Las Figuras (Serie Historia). Instituto Nacional de Antropología e Historia.

González, A. A. (1995). San Quintin: Comunidad pionera en la colonizacion de la Selva Lacandona [tesis de licenciatura, Universidad Autonoma Chapingo].

Gussinyer, J. (1976). Pinturas rupestres de Chiapas y el abrigo Juy-juy. Revista de la UNACH, 1, 79-102.

Gussinyer, J. (1980). Les pintures rupestres de l'abrie de Los Monos de Chiapas. Boletín Americanista, 22(30), $125-179$.

Künne, M., y Strecker, M. (eds.) (2008). Arte rupestre de México Oriental y América Central. Sociedad de Investigación del Arte Rupestre de Bolivia.

López de Llergo, G. (1925). Una visita a las ruinas de Yaxchilán, Alto Usumacinta. Memorias y Revista de la Sociedad Científica "Antonio Alzate", 44(9-12), 355-375.

Lozada, J. (2010). Espacio social y gráfica rupestre en la Sima del Copal, Ocozocoautla, Chiapas [tesis de licenciatura, Instituto Nacional de Antropología e Historia; Escuela Nacional de Antropología e Historia].

Lozada, J. (2017). El arte rupestre y la temporalidad del paisaje en Laguna Mensabak y Laguna Pethá, Chiapas [tesis de doctorado, Secretaría de Educación Pública; Instituto Nacional de Antropología e Historia; Escuela Nacional de Antropología e Historia].

Lozada, J. (2018). Informe Proyecto Arqueológico Pethá. Primera Temporada de Campo 2016. Consejo de Arqueología del Instituto Nacional de Antropología e Historia.

Machorro, R. (2005). Evaluación hidrogeológica de Alta Verapaz. Proyecto FODECYT 01-02. Consejo Nacional de Ciencia y Tecnología de Guatemala; Secretaría Nacional de Ciencia y Tecnología; Fondo Nacional de Ciencia y Tecnología.

Maler, T. (1903). Researches in the Central Portion of the Usumatsintla Valley: Report of Explorations for the Museum. Part Second. En Memoirs of the Peabody Museum of American Archaeology and Ethnology, 2(2).

Méndez, P. (1998). La Sima de las Cotorras. En D. Aramoni, T. Lee y M. Lisbona (coords.), Cultura y etnicidad zoque. Nuevos enfoques en la investigación social de Chiapas (pp. 47-61). Universidad de Ciencias y Artes de Chiapas; Universidad Autónoma de Chiapas.

Méndez, P. (1999). Climbing Prehistory Searching for the Painters of the Past. En G. Badino, A. Belotti, T. Bernabei et al. (eds.), Rio La Venta: Treasure of Chiapas (pp. 203-208). La Venta Associazione Culturale Esplorazioni Geografiche; Consejo Estatal para la Cultura y las Artes de Chiapas; Tipolitografia Turra.

Méndez, P. (2010). El Río La Venta y el Arco del Tiempo. La fascinante impronta del agua y el arco natural más alto del planeta, Chiapas, México. Gobierno del Estado de Chiapas; Talleres de Ink Servicios Gráficos.

Müllerried, F. K. G. (1957). Geología de Chiapas. Gobierno del Estado de Chiapas.

Navarrete, C. (1959). Exploraciones arqueológicas en la hacienda Las Palmas, Tecpatán, Chis. Revista ICACH, 3, 69-78.

Navarrete, C. (1966). The Chiapanec History and Culture (Paper 21). New World Archeological Foundation.

Navarrete, C. (1972). Fechamiento para un tipo de esculturas del sur de Mesoamérica. Anales de Antropología, IX, 45-52.

Navarrete, C. (1992). El hombre-danta en la iconografía el formativo superior de Chiapas y Guatemala. Instituto de Historia Natural de Chiapas.

Navarrete, C., y Martínez, E. (1961). Investigaciones arqueológicas en el Río Sabinal, Chiapas. Revista ICACH, 2(5), 49-83.

Navarrete, C., Lee, T. A., y Silva, C. (1993). Un catálogo de frontera. Esculturas, petroglifos y pinturas de la región media del Grijalva, Chiapas. Universidad Nacional Autónoma de México.

Ortega, F. et al. (1992). Carta geológica de la República mexicana. Instituto de Geología-Universidad Nacional Autónoma de México.

Palka, J. (2014). Maya Pilgrimage to Ritual Landscapes: Insights from Archaeology, Ethnohistory, and Ethnography (Archaeologies of Landscape in the Americas Series). University of New Mexico Press.

Palka, J., y Lozada, J. (2018). El periodo Posclásico en Chiapas y sus sitios arqueológicos más representativos. En Historia de Chiapas. Época prehispánica (pp. 89-120). Entretejas. 
Pincemin, S. (1999). De manos y soles: estudio de la gráfica rupestre en Chiapas. Universidad de Ciencias y Artes de Chiapas.

Rivero, S. E. (1985). Proyecto Miramar, Informe 1985. Primera temporada de campo. Consejo de Arqueología del Instituto Nacional de Antropología e Historia.

Rivero, S, E. (1988). Lacam-Tun: An achaeological site in the Lacandon Jungle, Chiapas, Mexico. En Recent Studies in Pre-Columbian Arrchaeology, part I (pp. 41-61). BAR International Series.

Rivero, S. E. (1989). Lacam-Tún, un sitio maya con historia. En Memorias del II Coloquio Internacional de Mayistas (pp. 1145-1156). Centro de Estudios Mayas-Universidad Nacional Autónoma de México.

Rivero, S. E. (1992). Laguna Miramar, Chiapas, México: una aproximación historica-arqueologica de los lacandones desde el clásico temprano. Instituto Chiapaneco de Cultura.

Scherer, A., y Golden, C. (2012). Revisiting Maler's Usumacinta: Recent Archaeological Investigations in Chiapas, Mexico (Monograph 1). Precolumbian Mesoweb Press.

Schmidt, H. J. (1979). Steinskulpturen vom Lago Miramar, Chiapas, Mexiko. Mexicon, I(5), 62-64.

Siffre, M. (1993). Découvertes dans les grottes mayas. Arthaud.

Stein, G. (1979). Ein Felsrelief am Lago Miramar, Chiapas, Mexico. Mexicon,I (5), 61-62.

Strecker, M. (1982). Representaciones de manos y pies en el arte rupestre de cuevas de Oxkutzcab, Yucatán. Boletín de la Escuela de Ciencias Antropológicas de la Universidad de Yucatán, 52, 47-57.

Strecker, M. (1987). Representaciones sexuales en el arte rupestre de la región maya. Mexicon, 9(2), 34-37.

Strecker, M. (2008). Arte rupestre de Tabasco y Chiapas. En M. Künne y M. Strecker (eds.), Arte rupestre de México Oriental y América Central. Sociedad de Investigación del Arte Rupestre de Bolivia.

Tello, C. (2004). En la Selva. Crónica de una viaje por la Lacandona. Joaquín Mortiz.

Unión Internacional para la Conservación de la Naturaleza, Instituto Nacional de Bosques (1999). Plan maestro del Parque Nacional Laguna Lachúa.

Weber, G., y Strecker, M. (1977) Die Petroglyphen der Finca Las Palmas, Chiapas, Mexico. Museum für Völkerkunde und Schweizerisches Museum für Volkskunde.

Woodfill, B. (2014). Three Previously Unrecorded Cave Features in the Western Highlands of Guatemala. Mexicon, 36(4), 114-120. https://bit.ly/2WPZcFi. 